\title{
EVALUACION DE LA BIOPSIA TRANSPEDICULAR GUIADA POR TAC
}

\author{
AVALIAÇÃO DA BIÓPSIA TRANSPEDICULAR GUIADA POR TC \\ EVALUATION OF TRANSPEDICULAR PERCUTANEOUS BIOPSY GUIDED BY CT
}

luis Miguel Rosales Olivarez ${ }^{1}$, Hugo Rolando Nieto Sandoval² ${ }^{2}$ Armando Alpízar Aguirre ${ }^{3}$, Barón Zárate Kalfopulos ${ }^{3}$,

María Guadalupe Sánchez Bringas ${ }^{3}$, Alejandro Antonio Reyes Sánchez ${ }^{4}$

\begin{abstract}
RESUMEN
Objetivo: Valorar la utilidad de la biopsia transpedicular percutánea guiada por Tomografía Axial Computarizada en conjunto con la sistematización de estudios como pruebas diagnósticas de la etiología de la destrucción vertebral. Métodos: Estudio de serie de casos prospectivo transversal de 21 pacientes a los que se les realizó biopsia transpedicular percutánea guiada por Tomografía Axial Computarizada y estudios de laboratorio y gabinete de marzo a julio del 2011, para evaluar su utilidad en el diagnóstico de destrucción vertebral. Resultados: Fueron 21 pacientes, 14 hombres y 7 mujeres, con edad media de 59,2 años, cuyos niveles más afectados estuvieron en L1, L2 y L3. El reporte de la biopsia tuvo una precisión diagnóstica del 90,4\%. En 2 casos se realizó correlación clínica entre biopsia y sistematización de estudios para obtener el diagnóstico. Conclusión: La biopsia guiada por Tomografía Axial Computarizada es una técnica sencilla, útil, de bajo costo y eficaz en el estudio de la destrucción vertebral; la sistematización de estudios permite corroborar el diagnóstico de la biopsia.
\end{abstract}

Descriptores: Columna vertebral; Enfermedades de la columna vertebral; Neoplasias de la columna vertebral; Biopsia; Estudios prospectivos.

\section{RESUMO}

Objetivo: Avaliar a utilidade da biópsia transpedicular percutânea guiada por tomografia axial computadorizada em conjunto com a sistematização de estudos, como exames diagnósticos da etiologia da destruição vertebral. Métodos: Estudo de série de casos, prospectivo e transversal de 21 pacientes submetidos à biópsia transpedicular percutânea guiada por tomografia axial computadorizada e exames laboratoriais e radiológicos, de março a julho de 2011, para avaliar sua utilidade no diagnóstico de destruição vertebral. Resultados: Foram analisados 21 pacientes, 14 homens e 7 mulheres, com média de idade de 59,2 anos, cujos níveis mais afetados foram L1, L2 e L3. O laudo da biópsia teve precisão diagnóstica de 90,4\%. Em dois casos, realizou-se a correlação clínica entre biópsia e sistematização de exames para obter o diagnóstico. Conclusão: A biópsia guiada por tomografia axial computadorizada é uma técnica simples, útil, de baixo custo e eficaz para o estudo da destruição vertebral; a sistematização de estudos permite corroborar o diagnóstico da biópsia.

Descritores: Coluna vertebral; Doenças da coluna vertebral; Neoplasias da coluna vertebral; Biopsia; Estudos prospectivos.

\section{ABSTRACT}

Objective: To evaluate the usefulness of percutaneous transpedicular biopsy guided by CT together with systematic studies such as diagnostic tests of the etiology of vertebral destruction. Methods: Case series, prospective and crossover study of 21 patients who underwent percutaneous transpedicular biopsy guided by CT and laboratory tests and radiological studies from March to July 2011, to evaluate its usefulness in the diagnosis of vertebral destruction. Results: We analyzed 21 patients, 14 men and 7 women, mean age 59.2 years, whose most affected levels were L1, L2, and L3. The biopsy report had diagnostic accuracy of $90.4 \%$. In 2 patients the clinical correlation between biopsy and systematization of diagnostic studies was carried out. Conclusion: CT-guided biopsy is a simple, useful, inexpensive, and effective technique for the study of vertebral destruction; the systematic studies allow us to corroborate the diagnosis made by biopsy.

Keywords: Spine; Spinal diseases; Spinal neoplasms; Biopsy; Prospective studies.

\section{INTRODUCCIÓN}

Muchas afecciones del sistema musculoesquelético se manifiestan como alteraciones focales, el cuerpo vertebral es un sitio común de lesiones ${ }^{1}$. El síndrome de destrucción vertebral se define como el conjunto de patologías que afectan la integridad estructural y modifican la arquitectura normal de la vértebra, produciendo inestabilidad raquídea y déficit neurológico ${ }^{2,3}$. Es una patología con etiología múltiple caracterizada por alteraciones de la estructura y ultraestructura en el hueso de la columna vertebral; que se acompaña de dolor e incapacidad funcional por alteraciones mecánicas y neurológicas ${ }^{2,3}$. Sus causas pueden ser infecciosas: bacterias, micobacterias, hongos y parásitos; tumores primarios: benignos y malignos; metástasis tumorales y enfermedades metabólicas: osteoporosis, osteomalacia, enfermedad de Paget, entre otras ${ }^{2,3}$. El diagnóstico de este síndrome está en relación con datos clínicos, estudios de laboratorio y gabinete. La resonancia magnética, tomografía axial computada, la fluoroscopia y la gammagrafía, suministran medios para ello. Otro elemento vital es la biopsia del cuerpo vertebral; ya que el diagnóstico preciso es esencial para definir el tratamiento oportuno y adecuado ${ }^{2,3}$. La columna vertebral es el tercer sitio más común para la presentación de la osteomielitis ${ }^{4}$. La etiología es por cualquier padecimiento que provoque bacteremia; la fuente más común son las infecciones de vías urinarias. Bacteriológicamente, a principios del siglo XX se reportaba casi exclusivamente el estafilococo dorado como agente patógeno ${ }^{5}$, actualmente, se han aislado otro tipo de microorganismos como los Gram negativos, Escherichia coli, Pseudomonas y Proteus ${ }^{4-6}$.

1. Jefatura de servicio de Cirugía de Columna Vertebral. Instituto Nacional de Rehabilitación (INR) - México, DF.

2. Curso de Alta Especialidad en Cirugía de Columna Vertebral. UNAM - México, DF

3. Servicio de Cirugía de Columna Vertebral, INR - México, DF.

4. División de Cirugía Especial, INR - México, DF.

Trabalho realizado Instituto Nacional de Rehabilitación (INR). Servicio de Cirugía de Columna. Mexico.DF.

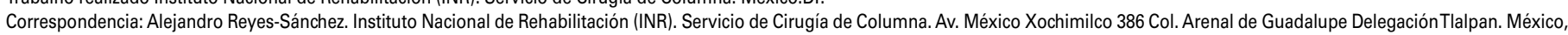
DF - CP 14389 - Email: alereyes@inr.gob.mx. 
Las neoplasias pueden originarse de las lesiones locales que se desarrollan dentro o adyacentes a la columna vertebral ó de neoplasias distantes ${ }^{5,7}$. La incidencia de neoplasias primarias de columna es de 2.5 a 8.5 por cada 100,000 persona al año ${ }^{8}$. Los tumores metastásicos son por mucho los más comunes; entre el 5 al 30\% de todos los pacientes con cáncer han tenido metástasis a la columna, predominando aquellos derivados de mama, pulmón, próstata o enfermedad linforreticular ${ }^{5,7-9}$. Las lesiones óseas sólo se pueden observar hasta que existe de un 30 a 50\% de destrucción trabecular ${ }^{9}$. Alpízar et al. ${ }^{3}$ propusieron una serie de exámenes de laboratorio y gabinete y, la biopsia, para optimizar recursos y llegar al diagnóstico etiológico del síndrome de destrucción vertebral; concluyéndose en el estudio que las pruebas con mayor sensibilidad y especificidad son: la resonancia magnética, la tomografía axial computada, la reacción en cadena de polimerasa para tuberculosis y la gammagrafía². El diagnóstico definitivo de estas patologías sólo es posible por biopsia. La biopsia abierta maximiza y asegura la obtención de tejido; sin embargo, la biopsia transpedicular percutánea guiada por imagenología ha mostrado ser muy segura ${ }^{10-13}$ y, puede ser realizada a todos los niveles de la columna ${ }^{2,5,11}$. Así, las biopsias se dividen en dos tipos: abierta y cerrada, percutánea o por punción. Un gran número de biopsias aún son abiertas, ya que se sigue considerando el procedimiento de elección ${ }^{14-16}$. La biopsia percutánea según la instrumentación utilizada y el tipo de muestra obtenida, se divide en punción-aspiración con aguja fina (BAAF) y en cilindro de tejido ${ }^{16}$. Es recomendable la realización de biopsias percutáneas en todas aquellas lesiones que se localicen en áreas anatómicas profundas, con una vía de acceso quirúrgica compleja y difícil, como lo es la columna vertebral e interior de la pelvis $^{14,15}$. La biopsia percutánea guiada por imagen se ha convertido en un procedimiento rutinario ${ }^{17}$, ya que presenta múltiples ventajas respecto a la biopsia abierta, tanto para el paciente como para el entorno sanitario. Entre ellas, es una cirugía ambulatoria con invasión mínima, más fácil de realizar, seguridad y ausencia de complicaciones relacionadas con la anestesia y la incisión quirúrgica, instauración pronta del tratamiento y reducción de $\operatorname{costos}^{14,16,18,19}$. Robertson e Ball20 en 1935 fueron los primeros en reportar el uso de biopsias percutáneas de la columna vertebral realizando punción-aspiración en 6 pacientes. Para 1956 Craig apud Ward e Kilpatrick ${ }^{15}$ desarrolló el uso de una trefina para la toma de biopsia vertebral. Hacia 1969 Ottolengui apud Biclels et al. ${ }^{21}$ sistematiza la biopsia vertebral percutánea para toda la columna toracolumbar. En 1986 Laredo e Bard ${ }^{22}$ describe la técnica percutánea guiada por fluoroscopia en 41 pacientes. Los primeros resultados satisfactorios se obtuvieron al utilizar métodos de imagen como guía, la fluroscopia concretamente ${ }^{23,24}$. El mérito de la utilización de la TAC para la realización de biopsia vertebrales se atribuye a Adapon et al. ${ }^{25}$ en 1981. En base al conocimiento y la experiencia adquirida con la instrumentación pedicular, se ha utilizado el pedículo como vía de abordaje en las biopsias, esta técnica disminuye el riesgo del abordaje paraespinal ${ }^{24,26,27}$. La técnica por vía transpedicular permite obtener muestras de tejidos más grandes, dependiendo del calibre de la aguja ${ }^{13}$. El uso de la biopsia por vía transpedicular guiada por TAC reporta resultados de seguridad diagnóstica entre el 70 al 100\%, con complicaciones menores al 5\% 2,28,29; permitiendo obtener el diagnóstico histológico cercano al $90 \%$ e identificar el agente causal de una infección en aproximadamente un 60\%; mientras que los pocos reportes de fluoroscopia refieren que el diagnóstico fue obtenido en un $55 \%{ }^{2,12}$. La biopsia guiada por TAC se utiliza para: determinar la naturaleza de una lesión en el tejido óseo con características inespecíficas, confirmar o descartar metástasis en pacientes con tumor primario maligno, excluir malignidad en la compresión del cuerpo vertebral, evaluar recurrencias después de una cirugía o del tratamiento médico, investigar fracturas patológicas y, estudiar y confirmar las infecciones musculoesqueléticas ${ }^{29}$. La precisión diagnóstica está influida por varios factores: tipo de aguja, lugar de la lesión, el tamaño de la muestra, la naturaleza de la lesión e institución donde se realiza la biopsia. Las fallas de este procedimiento pueden ser reducidas al mínimo si se realiza por manos experimentadas, con una evaluación cuidadosa previa del caso y enfoque en equipo ${ }^{29}$. Debido a lo anterior se establece como objetivo de del presunto estudio valorar la utilidad de la biopsia transpedicular percutánea guiada por tomografía axial computada en conjunto con la sistematización de estudios como pruebas diagnósticas de la etiología de la destrucción vertebral.

\section{MATERIAL Y MÉTODOS}

Estudio de serie de casos prospectivo transversal con tipo de muestreo no probabilístico de casos consecutivos de un universo de sujetos con diagnóstico de síndrome de destrucción vertebral en el periodo comprendido del primero de marzo al quince de julio del 2011, cuya muestra fue de 21 pacientes. Las personas estudiadas fueron de ambos géneros, mayores de 18 años, con protocolo de destrucción vertebral completo y que hayan firmado el consentimiento informado; no se incluyeron aquellos previamente diagnosticados y que contaran con alguna patología que les impidiera colocarse en decúbito prono para la toma de la biopsia y, se excluyeron a los que rechazaron la realización del procedimiento. De cada individuo se obtuvo la siguiente información: edad, sexo, segmento afectado, vértebra afectada, número de vértebras afectadas, hemoglobina, hematocrito, leucocitos, glucosa, urea, creatinina, tiempos de coagulación, exámen general de orina, proteína de Bence-Jones, VIH, reacción en cadena de polimerasa para tuberculosis, radiografías de cráneo, resonancia magnética nuclear, gammagrafía y medición del dolor posterior a la toma de la biopsia

Se realizó estadística descriptiva para determinar medias y desviaciones estándar o medianas y percentiles para las variables numéricas de acuerdo a su distribución. Así como frecuencias y porcentajes para las variables nominales. Se diseñaron gráficas y tablas de salida, utilizando el paquete estadístico Excel.

Técnica para la toma de biopsia. Cada paciente se presentó en el área de tomografía bañado, con ayuno de 8 horas y estudios de laboratorio prequirúrgicos. Ingresado a la sala tomográfica, se colocó en decúbito prono con los brazos extendidos sobre cama del tomógrafo, se realizó escaneo tomográfico con el aparato Light Speed VCT-64 cortes (GE, USA, 2007) de toda la columna vertebral con mayor énfasis en zona lesionada de acuerdo al diagnóstico de cada paciente. El radiólogo seleccionó y expuso en la pantalla el corte tomográfico a nivel de la zona de mayor destrucción vertebral, marcándose en piel la zona quirúrgica en la que se realizó la biopsia. En mesa de mayo sobre campos estériles abiertos, se colocaron instrumentos de trabajo estériles: aguja de Jamshidi 8G x $15.2 \mathrm{~cm}$ (Cardinal Health, República Dominicana, 2012), gasas, hoja de bisturí del número 15, flanera y martillo. Sobre el nivel y lado de mayor afectación vertebral, se infiltró 5 cc de lidocaína simple al $2 \%$ con aguja de $10 \mathrm{ml}$ a piel, celular subcutáneo, fascia y musculatura paravertebral; se incidió la piel $0.5 \mathrm{~cm}$ con hoja de bisturí, se insertó la aguja con punzón por lo planos mencionados hasta llegar a pedículo, se obtiene control tomográfico para verificar nivel y sitio de punción en vértebra, se pasa la aguja sin punzón a través del pedículo hasta el tercio anterior del cuerpo vertebral, aspiración del contenido de la lesión, se retira aguja, extrayéndose muestra de hueso obtenida de por lo menos $10 \mathrm{~mm}$, la cual se colocó en flanera y, se dividió para colocarse en frasco con formol para estudio histopatológico y reacción en cadena de polimerasa para tuberculosis y, en frasco con medio de Stuart para cultivo. Compresión de herida quirúrgica, control tomográfico, se coloca parche en herida y se regresa paciente a cama de traslado en decúbito prono, terminando el procedimiento. 


\section{RESULTADOS}

Se incluyeron 21 pacientes, 14 hombres (66.7\%) y 7 mujeres (33.3\%), con una edad media de 59.2 años (rango 28 a 82) presentándose la destrucción vertebral más frecuentemente en la sexta, séptima y octava décadas de la vida $(7,4$ y 4 casos respectivamente). Las vértebras lesionadas por paciente fueron 1 (13 casos), 2 (7 casos) y 3 (1 caso), por lo que 30 vértebras se afectaron en total; con mayor patología en la columna lumbar (16 casos, 53.3\%), siendo los niveles más afectados L1, L2 y L3 con 4 casos cada uno. En la columna torácica (10 casos, 33.3\%) los niveles patológicos fueron

T4 y T9 con 3 vértebras cada uno; la columna cervical y el sacro sólo presentaron un caso cada uno (Figura 1).

Los resultados y evaluación de los estudios de laboratorio en sangre no orientaron hacia alguna patología específica (Tabla 1).

El exámen general de orina fue patológico en 7 casos y se asoció a 5 casos de osteomielitis. La proteína de Bence Jones y la prueba de HIV fueron negativas en todos los casos y, la reacción en cadena de polimerasa para tuberculosis fue positiva en un caso, pero sin correlación a proceso infeccioso. Las radiografías de cráneo fueron negativas en búsqueda de lesiones líticas o blásticas en todos los pacientes. La resonancia magnética nuclear demostró lesiones compatibles a la patología que provocó la destrucción vertebral en 19 casos (90.4\%); así como la gammagrafía, que sólo en 2 casos no tuvo correlación con la lesión vertebral y fue diagnóstica en el 90.4\%. El reporte histopatológico de la biopsia correspondió a 6 diagnósticos, con una precisión diagnóstica en 19 muestras histopatológicas (90.4\%) con 2 casos de tejido óseo de aspecto normal; sólo 2 biopsias se reportaron como muestra inadecuada y no se presentaron casos de Mal de Pott. Al realizar la correlación clínica, el análisis de los estudios de laboratorio y gabinete y, el resultado de la biopsia, se integraron los diagnósticos finales (Figura 2). En las osteomielitis el cultivo reportó: estafilococo dorado (3), brucelosis

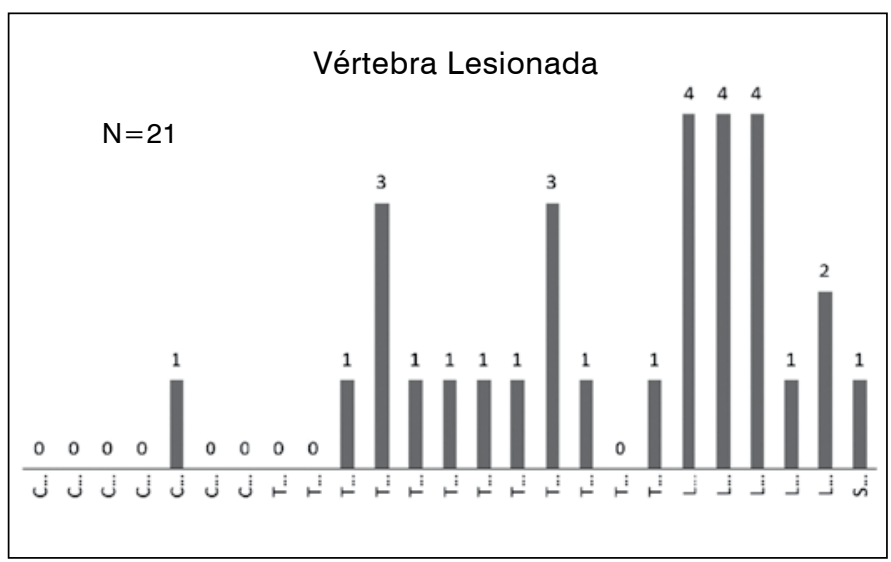

Figura 1. Nivel vertebral afectado.

Tabla 1. Estudios en sangre ( $\mathrm{N}=$ normal, $(+)$ arriba de rango, (-) por debajo de rango).

\begin{tabular}{c|c|c|c|c|c|c|c|c|c|c|c|c|c|c|c|c|c|c}
\hline & \multicolumn{3}{|c|}{ HB } & \multicolumn{3}{c|}{ HTC } & \multicolumn{3}{c|}{ LEUCOS } & \multicolumn{3}{c|}{ GLUC } & \multicolumn{3}{|c|}{ UREA } & \multicolumn{3}{|c}{ CRE } \\
\hline & $(+)$ & $\mathbf{N}$ & $(-)$ & $(+)$ & $\mathbf{N}$ & $(-)$ & $(+)$ & $\mathbf{N}$ & $(-)$ & $(+)$ & $\mathbf{N}$ & $(-)$ & $(+)$ & $\mathbf{N}$ & $(-)$ & $(+)$ & $\mathbf{N}$ & $(-)$ \\
\hline Osteomielitis & & 5 & 3 & & 5 & 3 & 1 & 7 & & 3 & 5 & & 1 & 7 & & & 7 & 1 \\
\hline Tumores & 1 & 2 & 3 & & 3 & 3 & 1 & 5 & & 1 & 5 & 1 & 1 & 5 & & & 6 & \\
\hline Metástasis & & 3 & & & 3 & & & 3 & & & 3 & & & 3 & & & 3 & \\
\hline Normal & & 1 & 1 & & 1 & 1 & 1 & 1 & & 1 & 1 & & & 2 & & & 2 & \\
\hline Inadecuada & 1 & 1 & & 1 & 1 & & 2 & & & 2 & & & 2 & & & 2 & \\
\hline
\end{tabular}

(1) y sin desarrollo (5). Los tumores fueron en 4 casos plasmocitoma y, con 1 cada uno: melanoma, linfoma no Hodking y ependimoma. El adenocarcinoma prostático fue el tumor primario que provocó un caso de metástasis. El dolor posterior a la toma de la biopsia tuvo una mediana y una moda de 7 (mínimo 3, máximo 10) y, no se presentaron complicaciones aparentes durante o posterior a la punción vertebral.

En los hombres la edad media fue 57.4 años (rango 28 a 76) con mayor afección en la sexta y séptima décadas de la vida con 4 casos respectivamente. La lesión vertebral se presentó más en la columna torácica con 10 casos, siendo los niveles más afectados T9 y T4 (3 y 2 casos respectivamente); la columna lumbar se lesionó en 9 ocasiones, principalmente a nivel de L1 (4 casos) y L2 (2 casos) y, la columna cervical y el sacro con una destrucción cada uno. El exámen general de orina fue normal en 11 pacientes y, hubo 3 patológicos, de los cuales 2 se asociaron a osteomielitis. La resonancia magnética nuclear mostró lesiones compatibles con el diagnóstico de la biopsia en 13 de 14 pacientes (92\%), el caso no certero reportó proceso infeccioso y el reporte histopatológico concluyó tumor. La gammagrafía fue captante en todas las lesiones. Los diagnósticos finales fueron los siguientes: osteomielitis 5 pacientes, 6 lesiones tumorales y 3 casos con metástasis (Figura 3). En la osteomielitis el cultivo

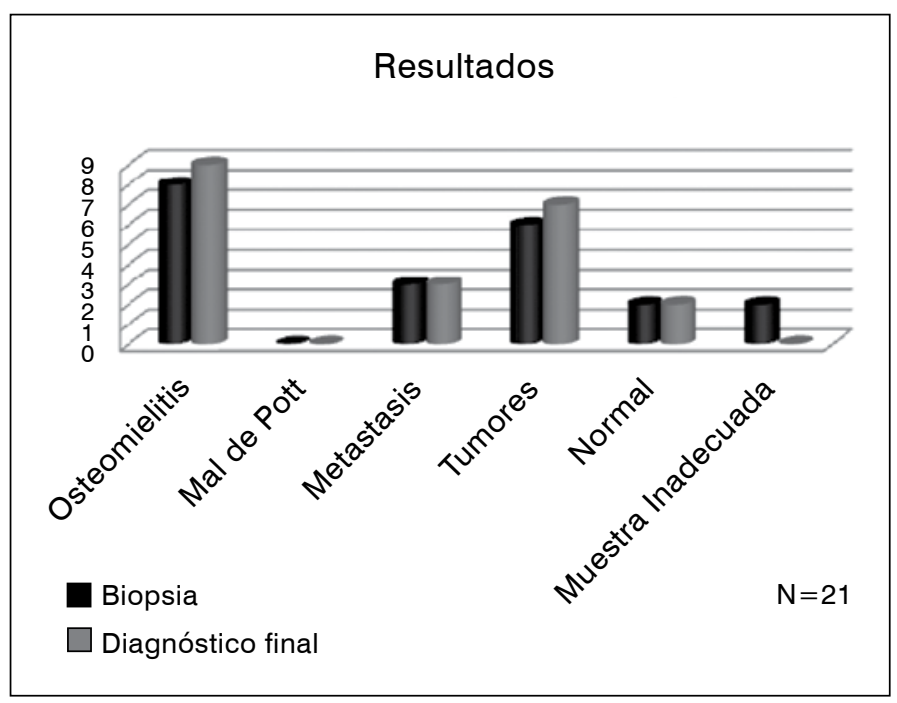

Figura 2. Diagnósticos.

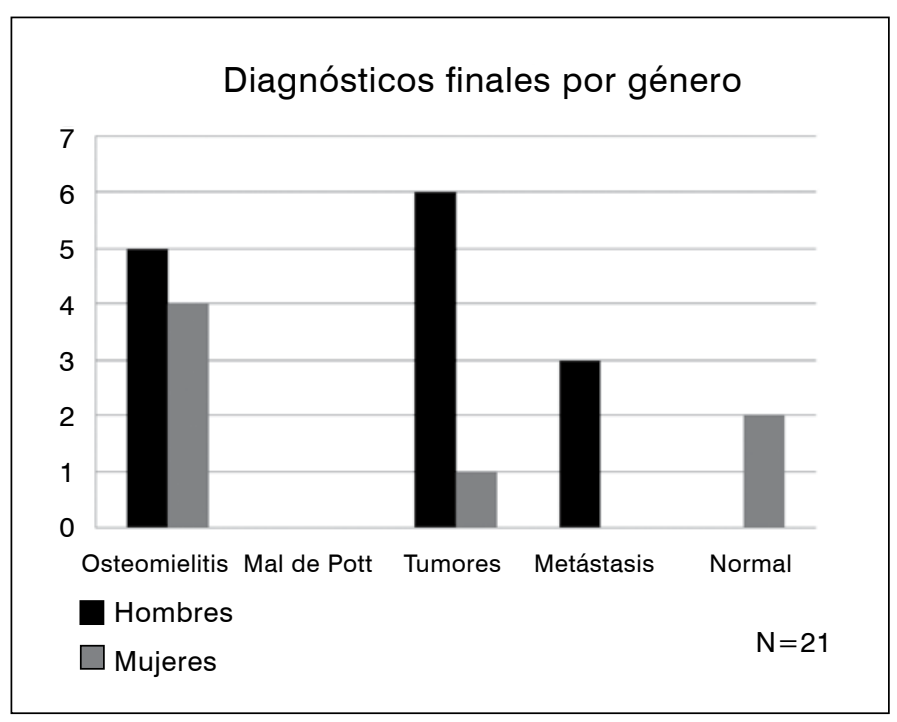

Figura 3. Comparación diagnóstica por género. 
se reportó así: 2 pacientes con estafilococo dorado, 1 caso de brucela y 1 no desarrolló germen causal. Los tumores reportados fueron: plasmocitoma 4 casos y, melanoma y linfoma no Hodking con un caso cada uno. Las células metastásicas provenían en uno de los casos del adenocarcinoma prostático. Por último, el dolor posterior a la biopsia tuvo una mediana y una moda de 6 (mínimo 3, máximo 9).

Para las mujeres la edad media fue 51.5 años (rango 40 a 82) con mayor afección en la sexta y octava décadas de la vida con 2 casos respectivamente. La lesión vertebral se presentó más en la columna lumbar con 7 casos, siendo los niveles más afectados L3 y L2 (3 y 2 casos respectivamente); la columna torácica se lesionó en 3 ocasiones a nivel de T4, T8 y T12. El exámen general de orina fue normal en 3 pacientes y, hubo 4 patológicos, de los cuales 3 se asociaron a osteomielitis. La resonancia magnética nuclear mostró lesiones compatibles con el diagnóstico de la biopsia en 6 de 7 pacientes (85\%), el caso no certero reportó proceso infiltrativo y el reporte histopatológico concluyó hueso normal con patrón degenerativo. La gammagrafía fue captante en todas las lesiones, aunque en 2 pacientes el diagnóstico final fue tejido normal. Los diagnósticos finales fueron los siguientes: osteomielitis 4 pacientes, 1 lesión tumoral (ependimoma) y 2 casos con tejido normal (Figura 3). En la osteomielitis el cultivo se reportó así: 1 paciente con estafilococo dorado y 3 no desarrollaron germen causal. Por último, el dolor posterior a la biopsia tuvo una mediana y una moda de 8 (mínimo 7, máximo 10).

\section{DISCUSIÓN}

La biopsia es la prueba clave en el diagnóstico de ciertas patologías osteomusculares. Tras una valoración clínica y radiológica, en la que no es posible obtener un diagnóstico, se indica la biopsia para precisar la patología y así, iniciar un tratamiento específico ${ }^{23}$. Una biopsia correcta es aquella que provee suficiente material para un diagnóstico correcto sin modificar el curso clínico ni el tratamiento ${ }^{13,14}$. La biopsia a cielo abierto se sigue considerando como la prueba de oro para la obtención de muestras, sin embargo, no está exenta a un resultado impreciso y está asociada en mayor número a complicaciones cutáneas ${ }^{1,14}$. Por lo que hay que recurrir a procedimientos que mantengan o aumenten la efectividad diagnóstica de la biopsia abierta, sean menos agresivos, de menor costo y con pocas complicaciones ${ }^{18}$. La técnica percutánea utilizando tomografía o fluroscopia como guía, ha reemplazado virtualmente el requerimiento de la biopsia quirúrgica abierta ${ }^{24}$. La biopsia transpedicular percutánea del cuerpo vertebral guiada por TAC ofrece un método mínimo invasivo para obtener muestras adecuadas de tejido y establecer un diagnóstico patológico 21,28 .

En nuestro estudio de 21 casos, semejante al de Yaffe et al. ${ }^{30}$ y Poyanli et al. ${ }^{31}$ (19 y 23 casos respectivamente) pero mayor que la serie de 9 pacientes de Hsu y Lim $^{32}$; se reporta más afección al sexo masculino como lo hacen De Lucas et al. ${ }^{33} \mathrm{y}$ Heyer et al. ${ }^{34}$. La edad promedio de presentación de nuestros pacientes es similar a la de Rivas et $a^{35}{ }^{35}$ y $\mathrm{Peh}^{36}$ y debajo de la media de Heyer et al. ${ }^{34}$ y Aribas et al. ${ }^{37}$. Ningún estudio revisado reporta el número de vértebras afectadas por paciente como está descrito en nuestra serie. Informamos que la columna lumbar presentó mayor patología coincidiendo con Rimondi et al. ${ }^{29}$ y Heyer et al. ${ }^{34}$. Este estudio mostró a los niveles L1, L2 y L3 como los más afectados al igual que Heyer et al. ${ }^{34}$ y Yaffe et al. ${ }^{30}$, aunque De Lucas et al. ${ }^{33}$ y Rimondi et al. ${ }^{29}$ reportan $L 4$ y L5 como los más lesionados. Rimondi et al. ${ }^{29}$ y Heyer et al. ${ }^{34}$ comentan que los estudios de laboratorio en sangre no son específicos para las patologías diagnosticadas en las vértebras lesionadas, al igual que nuestro reporte especificado en la Tabla
1; sólo algunas series refieren los tiempos de coagulación para poder realizar la toma de la biopsia. A todos nuestros pacientes se les realiza resonancia magnética nuclear para complementar el estudio radiológico, tal como lo efectúa Michel et al. ${ }^{38}$ en su estudio; en cambio Heyer et al. ${ }^{34}$ sólo lo realiza ante la sospecha de espondilitis y, Sehn e Gilula ${ }^{39}$ se basa en la presunción clínica y radiológica. La gammagrafía la utilizamos en búsqueda de lesiones a distancia del sitio lesionado, Rivas et al. ${ }^{35}$ prefiere realizarlo con TAC y, otros reportes sólo la realizan si hay sospecha clínica. La precisión diagnóstica de Poyanli et al. ${ }^{31}$ y Rivas et al. ${ }^{35}$ es semejante a la nuestra, sólo Yaffe et al. ${ }^{30}$ reporta certeza completa. Encontramos como patologías más frecuentes de la destrucción vertebral a la osteomielitis, los tumores y las metástasis; tal como lo reportan Rimondi et al. ${ }^{29}$, Rivas et al. ${ }^{35}$ y Poyanli et al. ${ }^{31}$. En el reporte infeccioso, Heyer et al. $^{34}$ y Sehn e Gilula ${ }^{39}$ precisan al estafilococo dorado como el principal germen detectado, al igual que nuestro estudio; pero, no detectamos germen causal en el $56 \%$ de los casos, como lo reportan Rimondi et al..$^{29}$ y De Lucas et al. ${ }^{33}$. Los principales tumores reportados en los casos de Rimondi et al. ${ }^{29}$ y Poyanli et al. ${ }^{31}$ que provocan la afección vertebral son: mieloma, plasmocitoma y linfoma; semejante a lo citado en este estudio. No reportamos complicaciones inherentes a la toma de la biopsia, tal como lo hace Yaffe et al. ${ }^{30}$; otros como Poyanli et al. ${ }^{31} \mathrm{y}$ Rimondi et al. ${ }^{29}$ presentan del 1-8\%. Nuestro estudio reportó y estadificó el dolor posterior a la punción, sólo Rivas et al. ${ }^{35}$ cita sin repercusiones valorables para el dolor.

Previamente en nuestro centro se había realizado un estudio para diagnosticar las lesiones que provocan la destrucción vertebral utilizando como guía el fluoroscopio, arrojando una precisión diagnóstica del 55\%, muy por debajo de lo reportado en ese momento en la literatura ${ }^{2}$. Debido a estos resultados poco precisos y no alentadores, se decidió implementar la biopsia transpedicular guiada por TAC para el estudio del síndrome de destrucción vertebral y así equiparar nuestros resultados con lo publicado actualmente.

Este estudio presenta menor número de pacientes por el tiempo en que fue realizado, pero los resultados son muy comparables con series de mayor número de pacientes y de tiempo de realización; ya que proporcionalmente se presenta en el mismo sexo, nivel de vértebras afectadas, reporte histopatológico, precisión diagnóstica, germen causal, tipo de tumores y tasa de complicaciones. Por eso consideramos que la biopsia transpedicular percutánea guiada por tomografía puede ser considerada de primera línea en nuestro medio para el diagnóstico de las lesiones que alteran el cuerpo vertebral.

\section{CONCLUSIONES}

La biopsia transpedicular percutánea guiada por tomografía axial computada es una técnica sencilla, útil, fiable, de bajo costo y eficaz; en el estudio de las lesiones destructivas de la columna vertebral.

La seguridad y los porcentajes en la precisión diagnóstica convierten a la biopsia transpedicular percutánea guiada por tomografía en una prueba electiva para obtener muestra de tejido suficiente e identificar el tipo de lesión que afecta la integridad estructural de las vértebras y así, establecer tratamientos específicos y eficaces para las patologías que provocan la destrucción vertebral.

En los pocos casos en los que el reporte de la biopsia es de muestra insuficiente o inespecífico, se utilizan como herramientas médicas: la correlación clínica y el análisis de las pruebas laboratorio y gabinete de la sistematización de estudios, que permiten establecer un diagnóstico. 


\section{REFERENCIAS}

1. Stoker DJ. Skeletal radiology: Merit Communications. Capt Bone Biopsy. 1992. p. 81-92.

2. Rosales LM, Valle I, Alpízar A, Miramontes V, Reyes A. Evaluación de la biopsia percutánea en el diagnóstico del síndrome de destrucción torácico y lumbar. Cir Ciruj. 2007;75:459-63.

3. Alpízar A, Elías A, Rosales LM, Miramontes V, Reyes A. Síndrome de destrucción vertebral. Sistemas de evaluación en su diagnóstico. Cir Ciruj. 2008;76:205-11.

4. Jaramillo-de la Torre JJ, Bohinski RJ, Kuntz C 4th. Vertebral osteomyelitis. Neurosurg Clin N Am. 2006;17(3):339-51

5. Balderston RA. Afflictions of the vertebrae. In: Herkowitz HN, Garfin SR, Eismont FJ, Bell GR. Rothman-Simeone the Spine. 5th ed. Philadelphia: Saunders/Elsevier; 2006. p. 1233-354.

6. Lew DP, Waldvogel FA. Osteomyelitis. N Engl J Med. 1997;336(14):999-1007.

7. Simmons ED, Zheng Y. Vertebral tumors: surgical versus nonsurgical treatment. Clin Orthop Relat Res. 2006;443:233-47.

8. Khan SN, Donthineni R. Surgical management of metastatic spine tumors. Orthop Clin North Am. 2006;37(1):99-104.

9. Bloomer CW, Ackerman A, Bhatia RG. Imaging for spine tumors and new applications. Top Magn Reson Imaging. 2006:17(2):69-87.

10. Hadjipavlou AG, Kontakis GM, Gaitanis JN, Katonis PG, Lander P, Crow WN. Effectiveness and pitfalls of percutaneous transpedicle biopsy of the spine. Clin Orthop Relat Res. 2003;(411):54-60.

11. Pierot $L$, Boulin A. Percutaneous biopsy of the thoracic and lumbar spine: transpedicular approach under fluoroscopic guidance. AJNR Am J Neuroradiol. 1999;20(1):23-5.

12. Krause ND, Haddad ZK, Winalski CS, Ready JE, Nawfel RD, Carrino JA. Musculoskeletal biopsies using computed tomography fluoroscopy. J Comput Assist Tomogr. 2008;32(3):458-62

13. Nourbakhsh A, Grady JJ, Garges KJ. Percutaneous spine biopsy: a meta-analysis. J Bone Joint Surg Am. 2008:90(8):1722-5.

14. Simon MA, Biermann JS. Biopsy of bone and soft-tissue lesions. J Bone Joint Surg Am. 1993;75(4):616-21

15. Ward WG Sr, Kilpatrick S. Fine needle aspiration biopsy of primary bone tumors. Clin Orthop Relat Res. 2000;(373):80-7.

16. Heare TC, Enneking WF, Heare MM. Staging techniques and biopsy of bone tumors. Orthop Clin North Am. 1989;20(3):273-85

17. Gangi A. Interventional musculoskeletal radiology. Eur Radiol. 2002;12(6):1235-6.

18. Ruhs SA, el-Khoury GY, Chrischilles EA. A cost minimization approach to the diagnosis of skeletal neoplasms. Skeletal Radiol. 1996;25(5):449-54.

19. Stoker DJ, Kissin CM. Percutaneous vertebral biopsy: a review of 135 cases. Clin Radiol. 1985;36(6):569-77

20. Robertson RC, Ball RP. Destructive spinal lesions: diagnosis by needle biopsy. J Bone Joint Surg. 1935:17:749-58.

21. Bickels J, Jelinek JS, Shmookler BM, Neff RS, Malawer MM. Biopsy of musculoskeletal tumors. Current concepts. Clin Orthop Relat Res. 1999;(368):212-9.
22. Larédo JD, Bard M. Thoracic spine: percutaneous trephine biopsy. Radiology. 1986:160(2):485-9

23. Bender CE, Berquist TH, Wold LE. Imaging-assisted percutaneous biopsy of the thoracic spine. Mayo Clin Proc. 1986;61(12):942-50.

24. Hadjipavlou A, Gaitanis I, Crow W, Lander P, Katonis P, Kontakis G. The results, effectiveness and pitfalls of the percutaneous transpedicular biopsy (PTB) of the thoracic and lumbar spine. J Bone Joint Surg Br. 2004;86(Suppl 3):370-1.

25. Adapon BD, Legada BD Jr, Lim EV, Silao JV Jr, Dalmacio-Cruz A. CT-guided closed biopsy of the spine. J Comput Assist Tomogr. 1981;5(1):73-8.

26. Metzger CS, Johnson DW, Donaldson WF 3rd. Percutaneous biopsy in the anterior thoracic spine. Spine (Phila Pa 1976). 1993;18(3):374-8.

27. Gaitanis I, Crow W. Percutaneous transpedicular biopsy of the spine. J Bone Joint Surg Br. $2001 ; 83($ Suppl):238

28. Dave BR, Nanda A, Anandjiwala JV. Transpedicular percutaneous biopsy of vertebral body lesions: a series of 71 cases. Spinal Cord. 2009:47(5):384-9.

29. Rimondi E, Rossi G, Bartalena T, Ciminari R, Alberghini M, Ruggieri $P$, et al. Percutaneous CT-guided biopsy of the musculoskeletal system: results of 2027 cases. Eur J Radiol. 2011;77(1):34-42.

30. Yaffe D, Greenberg G, Leitner J, Gipstein R, Shapiro M, Bachar GN. CT-guided percutaneous biopsy of thoracic and lumbar spine: A new coaxial technique. AJNR Am J Neuroradiol. 2003;24(10):2111-3.

31. Poyanli O, Akan K, Unay K, Tangay C. CT-guided percutaneous transpedicular biopsy for the diagnosis of vertebral lesions. Acta Orthop Belg. 2008;74(4):503-6.

32. Hsu WC, Lim KE. Computed tomography-guided percutaneous transpedicular biopsy of the thoracic spine. Chang Gung Med J. 2001:24(6):368-75

33. de Lucas EM, González Mandly A, Gutiérrez A, Pellón R, Martín-Cuesta L, Izquierdo J, et al. CT-guided fine-needle aspiration in vertebral osteomyelitis: true usefulness of a common practice. Clin Rheumatol. 2009:28(3):315-20.

34. Heyer CM, Brus LJ, Peters SA, Lemburg SP. Efficacy of CT-guided biopsies of the spine in patients with spondylitis--an analysis of 164 procedures. Eur J Radiol. 2012;81(3):e244-9.

35. Rivas A, Cáceres E, Ubierna M. Reliability, sensitivity, specificity and safety of computed tomography-guidede percutaneous vertebral biopsies. Rev Ortop Traumatol (Madr) 2007:51:245-55

36. Peh W. CT-guided percutaneous biopsy of spinal lesions. Biomed Imaging Interv J. 2006;2(3):e25

37. Aribas BK, Dingil G, Dogan K, Sahin G, Pak I, Ardic F. Factors in sample volume and quality of CT-guided vertebral biopsy: location and needle trajectory. Turk Neurosurg. 2010;20(1):21-6.

38. Michel SC, Pfirmann CW, Boos N, Hodler J. CT-guided core biopsy of subchondral bone and intervertebral space in suspected spondylodiskitis. AJR Am J Roentgenol. 2006:186(4):977-80

39. Sehn JK, Gilula LA. Percutaneous needle biopsy in diagnosis and identification of causative organisms in cases of suspected vertebral osteomyelitis. Eur J Radiol. 2012;81(5):940-6. 